\section{A limited role of cytokine storm and fibrogenesis in COVID-19 related liver injury}

\section{To the Editor,}

Symptomatic transaminases elevations are common in coronavirus disease 2019 (COVID-19). In approximately $23 \%$ of all patients elevated transaminases are seen. COVID-19 related liver injury may be caused by primary liver injury but also as collateral damage related to drug-induced-liver-injury, hypoxia, and preexisting liver disease [1]. Hepatitis may aggravate disease severity, and lead to mortality. In this context, it is important to understand the impetus for liver injury [2].We performed a multimodality approach to dissect the contributing factors to liver injury in a well-defined cohort of COVID-19 patients. The aim was to explore inflammatory and fibrosis biomarkers and their relation to pre-existing liver disease.

We examined a cohort of 25 patients (mean age 57.9 years, male $72 \%$, median body mass index $27.3 \mathrm{~kg} / \mathrm{m}^{2}$ ) who were hospitalized in 2020 in the Radboudumc, Nijmegen, The Netherlands. All presented with COVID-19 infection established on basis of a positive polymerase chain reaction
(PCR) test and/or typical findings on thorax computed tomography (CT). We collected clinical data on the disease course of COVID-19 during hospitalization, as well as from the medical history (Table I). Liver stiffness was assessed using ElastPQ point shear wave elastography and liver steatosis was determined using computer assisted ultrasound [3]. Routine clinical laboratory parameters were measured at multiple time points during hospitalization. Inflammatory cytokines were measured with a commercially available multiplex proximity extension assay (Olink proteomics AB, Uppsala, Sweden) [4].

Liver injury defined as the difference between the highest (maximum) and lowest (minimum) alanine aminotransferase (delta ALAT) that exceeded twice the upper limit of normal (90 U/L), developed in 11/25 patients (Fig. 1), and 9/25 (36\%) required admission to the intensive care unit (ICU). Liver injury was associated with ICU admission $(63.6 \%$ vs $14.3 \%$ $\mathrm{p}=0.017)$ and mechanical ventilation (63.6\% vs $7.1 \% \mathrm{p}=0.007)$. Liver stiffness was independent of liver injury $(4.7 \pm 1.5 \mathrm{kPa}$ vs $4.7 \pm 2.3 \mathrm{kPa}, \mathrm{n}=24, \mathrm{p}=0.728)$ or of steatosis $(7.1 \pm 4.5$ vs $6.9 \pm 4.3, \mathrm{n}=22 \mathrm{p}=0.973$ ). In this study, liver stiffness values were within normal ranges and independent of (degree of)
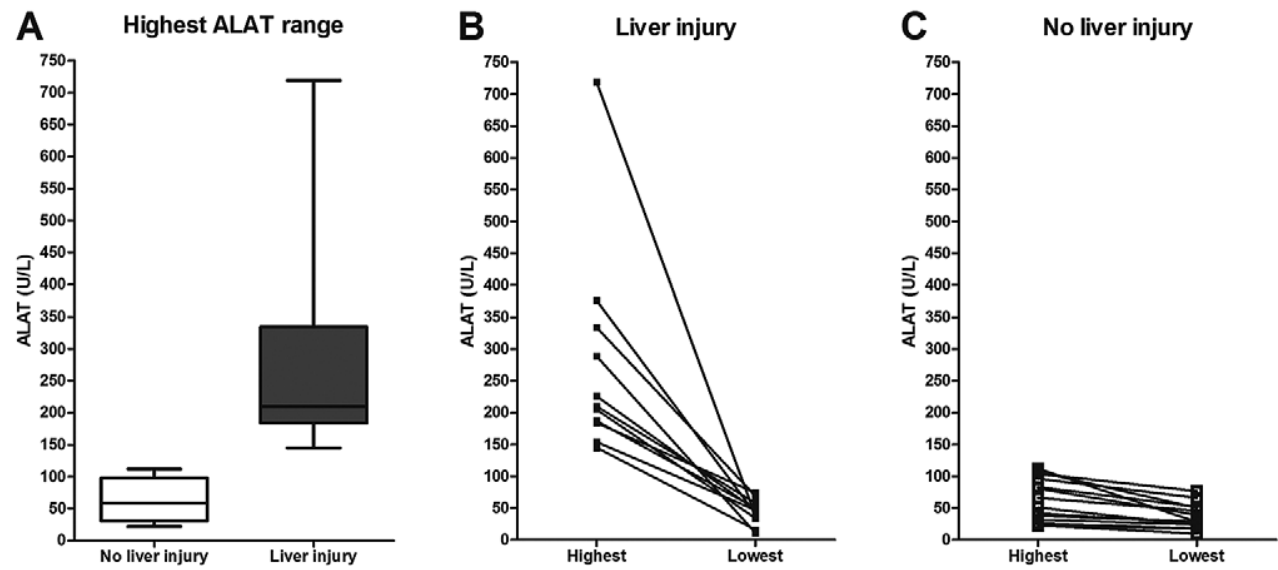

Fig. 1. Overview of liver injury markers. A: Boxplot showing range of highest alanin aminotransferase (ALAT) measured grouped by patients with liver injury (a delta ALAT during admission of $>90 \mathrm{U} / \mathrm{L}$ ) and without liver injury $(<90 \mathrm{U} / \mathrm{L})$. B: highest and lowest ALAT measured during admission per patient in the group with liver injury. C: Highest and lowest ALAT measured during admission per patient in the group without liver injury. 
Table I. Laboratory parameters, elastography outcomes, inflammation and fibrosis markers of liver injury versus no liver injury group

\begin{tabular}{|c|c|c|c|c|c|}
\hline Liver injury markers & $\mathrm{n}$ & All patients & $\begin{array}{l}\text { Patients without } \\
\text { liver injury }\end{array}$ & $\begin{array}{l}\text { Patients with } \\
\text { liver injury }\end{array}$ & $\mathrm{p}$ \\
\hline Highest ALAT & 25 & $156.5 \pm 153.1$ & $63.1 \pm 33.6$ & $275.4 \pm 164.4$ & N.A. \\
\hline Lowest ALAT & 25 & $39.6 \pm 19.6$ & $36.1 \pm 19.6$ & $44.1 \pm 19.5$ & 0.317 \\
\hline ASAT (U/L) & 19 & $143.6 \pm 114.1$ & $58.9 \pm 25.5$ & $219.8 \pm 108.8$ & 0.0004 \\
\hline GGT (U/L) & 20 & $272.3 \pm 313.1$ & $143.4 \pm 241.0$ & $429.8 \pm 330.8$ & 0.007 \\
\hline $\begin{array}{l}\text { Alkaline phosphatase } \\
\text { (U/L) }\end{array}$ & 18 & $162.9 \pm 106.3$ & $125.0 \pm 88.3$ & $200.9 \pm 113.9$ & 0.05 \\
\hline Bilirubin total $(\mu \mathrm{mol} / \mathrm{L})$ & 21 & $11.1 \pm 6.9$ & $11.9 \pm 7.9$ & $10.3 \pm 5.9$ & 0.557 \\
\hline \multicolumn{6}{|l|}{ Elastography/steatometry } \\
\hline Liver stiffness (kPa) & 24 & $4.7 \pm 1.9$ & $4.7 \pm 2.3$ & $4.7 \pm 1.5$ & 0.728 \\
\hline Steatosis (\%) & 22 & $7.0 \pm 4.3$ & $7.1 \pm 4.5$ & $6.9 \pm 4.3$ & 0.973 \\
\hline \multicolumn{6}{|l|}{ Inflammation markers } \\
\hline CRP (mg/L) & 24 & $174.0 \pm 98.3$ & $153.3 \pm 91.24$ & $203.0 \pm 105.3$ & 0.198 \\
\hline Ferritin $(\mu \mathrm{mol} / \mathrm{L})$ & 24 & $1644.7 \pm 2023.1$ & $1083.3 \pm 906.3$ & $2308.1 \pm 2744.8$ & 0.087 \\
\hline D-dimer (ng/ml) & 23 & $8802.6 \pm 23507.1$ & $1992.8 \pm 1889.6$ & $\begin{array}{c}19395.6 \pm \\
36144.4\end{array}$ & 0.378 \\
\hline G-CSF (pg/ml) & 21 & $79.2 \pm 58.2$ & $76.4 \pm 56.8$ & $82.3 \pm 62.6$ & 0.463 \\
\hline IL-6 (pg/ml) & 21 & $152.7 \pm 325.6$ & $93.2 \pm 127.6$ & $218.2 \pm 456.5$ & 0.458 \\
\hline IP-10 (pg/ml) & 21 & $1420.2 \pm 1289.1$ & $1385.4 \pm 1467.5$ & $1458.5 \pm 1138.7$ & 0.526 \\
\hline MCP-1 (pg/ml) & 21 & $662.8 \pm 610.5$ & $481.6 \pm 475.2$ & $862.1 \pm 702.1$ & 0.091 \\
\hline TNF- $\alpha(\text { NPX })^{*}$ & 20 & $3.3 \pm 0.5$ & $3.2 \pm 0.3$ & $3.6 \pm 0.6$ & 0.021 \\
\hline IL-18 (NPX)* & 20 & $9.0 \pm 0.2$ & $8.7 \pm 0.1$ & $9.5 \pm 3.5$ & 0.014 \\
\hline IL-10 (NPX)* & 20 & $4.9 \pm 1.2$ & $4.7 \pm 1.1$ & $5.1 \pm 1.5$ & 0.589 \\
\hline \multicolumn{6}{|l|}{ Liver fibrosis markers } \\
\hline P3NP $(\mu g / L)$ & 23 & $7.9 \pm 4.6$ & $5.9 \pm 1.5$ & $10.6 \pm 5.9$ & 0.002 \\
\hline TGF- $\beta($ NPX)* & 20 & $7.4 \pm 0.8$ & $7.3 \pm 0.7$ & $7.7 \pm 0.9$ & 0.165 \\
\hline MMP-1 (NPX)* & 20 & $11.2 \pm 1.5$ & $11.1 \pm 1.5$ & $11.3 \pm 1.6$ & 0.877 \\
\hline FGF-21 (NPX)* & 20 & $5.7 \pm 2.3$ & $5.8 \pm 2.2$ & $5.6 \pm 2.5$ & 0.817 \\
\hline HGF $(\mathrm{NPX})^{*}$ & 20 & $9.7 \pm 1.1$ & $9.1 \pm 0.7$ & $10.5 \pm 1.2$ & 0.005 \\
\hline
\end{tabular}

ALAT: alanine aminotransferase; ASAT: aspartate aminotransferase; GGT: gamma glutamyl transferase; CRP: C reactive protein; G-CSF: granulocyte colony-stimulating factor; IL: interleukine; IP10: InterferonInducible Protein 10; MCP-1: monocyte chemoattractant protein-1; TNF- $\alpha$ : tumor necrosis factor alpha; P3NP: amino-terminal pro-peptide of type III procollagen; TGF- $\beta$ : transforming growth factor beta; MMP-1: matrix metalloproteinase-1; FGF-21: Fibroblast growth factor 21; HGF: hepatic growth factor; ${ }^{*}$ results of proximity extension assays expressed in normalized protein expressions, which is a log-2 arbitrary unit, that only allows for relative quantification inside this cohort.

liver injury suggesting the absence of significant pre-existing fibrosis. Patients with liver injury had significantly higher value of the fibrogenesis marker amino-terminal pro-peptide of type III procollagen (P3NP) (10.6 \pm 5.9 vs. $5.9 \pm 1.5 \mathrm{ng} / \mathrm{mL}, \mathrm{p}$ $=0.002$ ), and overall, there was a robust correlation between ALAT and P3NP $(r=0.773, p<0.01)$. Hepatic growth factor (HGF) was increased in patients with liver injury in contrast to transforming growth factor beta (TGF- $\beta$ ), a key regulator of fibrogenesis. As the liver injury group contained 7 patients that underwent mechanical ventilation, $\mathrm{P} 3 \mathrm{NP}$ might pertain more to fibroproliferation in the lungs during acute respiratory distress syndrome. HGF induces breakdown of extracellular matrix but is mainly implicated in enhancing liver regeneration after liver injury.

We found that the inflammatory cytokines: interleukin (IL) 6 , granulocyte colony-stimulating factor (G-CSF), interferon- inducible protein 10 (IP10), monocyte chemoattractant protein-1 (MCP-1) were elevated in the complete cohort, but there was no interaction with liver injury. Tumor necrosis factor alpha (TNF- $\alpha)(\mathrm{p}=0.021)$ and IL-18 $(\mathrm{p}=0.014)$ were higher in patients with liver injury, but overall, we could not demonstrate a dominant pro-inflammatory profile. Collectively our data suggest that the cytokine pattern has a limited role in COVID-19 related liver injury.

Our steatometry results show that steatosis is not overrepresented in patients with liver injury. Interestingly, patients with liver injury lacked increased FGF-21 expression. FGF-21 is an insulin independent regulator of glucose and lipid metabolism, elevated in the plasma of patients with simple steatosis or steatohepatitis [5].

In summary, the current study shows that liver injury, as expressed by elevated ALAT values are ubiquitous in 
COVID-19. There is a limited role of pro-inflammatory cytokines and fibrogenesis in the pathogenesis of liver injury.

Menso J. Westerouen van Meeteren ${ }^{1}$, Frederik M.A. van den Heuvel $^{2}$, Gert Weijers ${ }^{3}$, Leo A.B. Joosten ${ }^{3}$, Quirijn de Mast $^{3}$, Frank L. van de Veerdonk ${ }^{3}$, Peter Pickkers ${ }^{4}$, Aline H. de Nooijer ${ }^{3}$, Wouter Hoefsloot $^{5}$, Joost P.H. Drenth ${ }^{1}$, Chris L. de Korte ${ }^{6}$, Robin Nijveldt ${ }^{2}$, Mihai G. Netea ${ }^{3}$, Eric T.T.L. Tjwa ${ }^{1}$, on behalf of the Radboudumc COVID-19 study group

1) Department of Gastroenterology and Hepatology, Radboud University Medical Center, Nijmegen; 2) Department of Cardiology, Radboud University Medical Center, Nijmegen; 3) Department of Internal Medicine, Radboud University Medical Center, Nijmegen; 4) Department of Intensive Care Medicine, Radboud University Medical Center, Nijmegen 5) Department of Pulmonary Disease, Radboud University Medical Center, Nijmegen; 6) Department of Radiology, Radboud University Medical Center, Nijmegen, The Netherlands

Correspondence: Eric T.T.L. Tjwa, eric.tjwa@radboudumc.nl

\section{Conflicts of interest: None.}

Acknowledgements: MGN is supported by a Spinoza grant of the Netherlands Organization for Scientific Research, and an ERC Advanced Grant (\#833247).

DOI: $10.15403 /$ jgld-3262

\section{REFERENCES}

1. Schattenberg JM, Labenz C, Wörns MA, et al. Patterns of liver injury in COVID-19 - a German case series. United European Gastroenterol J 2020;8:814-819. doi:10.1177/2050640620931657

2. Alqahtani SA, Schattenberg JM. Liver injury in COVID-19: The current evidence. United European Gastroenterol J 2020;8:509-519. doi:10.1177/2050640620924157

3. Weijers G, Wanten G, Thijssen JM, van der Graaf M, de Korte CL. Quantitative Ultrasound for Staging of Hepatic Steatosis in Patients on Home Parenteral Nutrition Validated with Magnetic Resonance Spectroscopy: A Feasibility Study. Ultrasound Med Biol 2016;42:637644. doi:10.1016/j.ultrasmedbio.2015.11.004

4. van de Veerdonk FL, Janssen NAF, Grondman I, et al. A systems approach to inflammation identifies therapeutic targets in SARS-CoV-2 infection. medRxiv 2020. doi:10.1101/2020.05.23.20110916

5. Liu J, Xu Y, Hu Y, Wang G. The role of fibroblast growth factor 21 in the pathogenesis of non-alcoholic fatty liver disease and implications for therapy. Metabolism 2015;64:380-390. doi:10.1016/j. metabol.2014.11.009

\section{GAVE: a gastroenterologist challenge}

\section{To the Editor,}

We read with interest the article of Pioppo et al. [1] in which, starting from an impressive number $(18,375)$ of cases with index admissions for GAVE the authors reported a high proportion $(20.49 \%)$ of readmissions within 30 days, and identified two risk factors for readmission: the presence of portal hypertension and of chronic kidney disease. Since the main cause for readmission in the aforementioned study was rebleeding, one can speculate that GAVE targeted endoscopic treatment at the first admission might reduce the rebleeding rate and consequently the number of readmissions.

The optimal treatment of GAVE is currently not established. A number of endoscopic options are available: argon plasma coagulation (APC), radiofrequency ablation (RFA) and band ligation (BL). While RFA is not widely available, APC and $\mathrm{BL}$ are easily accessible. The data in literature favoring one or the other of these methods is hard to interpret due to the variability of the extent of treatment performed (complete or near eradication of GAVE), due to various definitions of treatment success and also due to the duration of follow up [2]. While some authors found no difference between methods [3], others reported a higher rebleeding rate after APC and better outcome for $\mathrm{BL}$, patients with previous failure to APC responding to $\mathrm{BL}$ [4]. An important factor to consider might be the endoscopic appearance of GAVE (three types are described: linear-watermelon stomach, punctate and nodular) and the particular treatment applied in each form since, for example, better outcomes are reported with BL for the nodular type [5].

We retrospectively evaluated all patients admitted to our unit with GAVE who had received endoscopic treatment in the last $2 \frac{1}{2}$ years, with a minimum follow up period of 6 months, and a median follow up of 18 (0-25) months. Seventeen patients were included, mean age $69.82 \pm 8.83$ years, $58.8 \%$ females, $94.1 \%$ with at least one comorbidity $(58.8 \%$ with liver cirrhosis, and $35.28 \%$ with other comorbidities: valvular or ischemic heart disease, diabetes mellitus, hypertension). Thirteen received APC and 5 received BL; one patient with rebleeding after APC responded to $\mathrm{BL}$. Complete eradication of lesions was aimed at each endoscopic session, the median time elapsed between sessions being one month. We observed a trend towards multiple sessions for APC with a median number of 3 (1-10) as compared with 2 (1-2) in the BL group. Unplanned readmissions were necessary for overt rebleeding in 6 patients $(35.3 \%)$ and occurred after a median of 55 (30144) days after the index admission, only two (11.7\%) being early admissions (in the first 30 days). Overall, after endoscopic treatment the median increase of hemoglobin level was $2 \mathrm{~g} /$ dl (2.6-4.7).

From our experience, endoscopic treatment for GAVE is effective in reducing acute rebleeding and chronic blood loss, as seen from the low number of early unplanned readmissions and respectively from the improvement in the hemoglobin level. Though, in the long term the rebleeding rate rises so we consider that the improvement of the efficacy of endoscopic treatment is indicated. The optimal type (APC vs BL vs combination of both) and timing of endoscopic treatment still remains to be determined and for this, due to the small number of GAVE cases even in tertiary gastroenterology clinics, multicentric studies are necessary.

\section{Roxana Vadan, Stefania Bunduc, Liana Gheorghe, Cristian Gheorghe}

Gastroenterology and Hepatology Center, Fundeni Clinical Institute, Carol Davila University of Medicine and Pharmacy, Bucharest, Romania 
Correspondence: Roxana Vadan, roxanavadan@yahoo.com

Conflicts of interest: None.

DOI: $10.15403 /$ jgld-2921

\section{REFERENCES}

1. Pioppo L, Bhurwal A, Raj Mutneja H, et al. Portal Hypertension and Chronic Kidney Disease Significantly Increase the Risk of Early Unplanned Readmission in GAVE-Related Admissions. J Gastrointestin Liver Dis 2020;29:151-157. doi:10.15403/jgld-804

2. Zepeda-Gomez S. Endoscopic treatment for Gastric Antral Vascular Ectasia: Current Options. GE Port J Gastroenterol 2017;24:176-182. doi:10.1159/000453271

3. St Romain P, Boyd A, Zheng J, Chow SC, Burbridge R, Wild D. Radiofrequency ablation (RFA) vs argon plasma coagulation (APC) for the management of gastric antral vascular ectasia (GAVE) in patients with and without cirrhosis: results from a retrospective analysis of a large cohort of patients treated at a single center. Endosc Int Open 2018;6:E266-E270. doi:10.1055/s-0043-123187

4. Elhendawy M, Mosaad S, Alkhalawany W, et al. Randomized controlled study of endoscopic band ligation and argon plasma coagulation in the treatment of gastric antral and fundal vascular ectasia. United European Gastroenterol J 2016;4:423-428. doi:10.1177/2050640615619837

5. Wright AP, Mellinger JL, Prabhu A. Stepwise endoscopic eradication of refractory nodular gastric antral vascular ectasia by use of detachable snare and band ligation. VideoGIE 2016;2:4-5. doi:10.1016/j. vgie.2016.11.004

\section{A spontaneous rupture of lung metastasis from hepatocellular carcinoma after the introduction of lenvatinib}

\section{To the Editor,}

We herein present a case of a spontaneous rupture of lung metastasis from hepatocellular carcinoma (HCC) after the introduction of lenvatinib. A 71-year-old man known with alcoholic hepatitis was referred to our hospital for a liver tumor workup. He was diagnosed with HCC with multiple lung metastases. His Child-Pugh score was A5, and he was started on oral lenvatinib as palliative chemotherapy. Seven days after the introduction of lenvatinib, he experienced persistent chest pain. Chest radiography showed pleural effusion and an increased pulmonary opacity in the right lung. Contrastenhanced computed tomography (CT) revealed a ruptured tumor with hemothorax in the right lung (Fig. 1). Emergency angiography showed contrast extravasation from the right bronchial artery, and bronchial artery embolization was therefore performed. There was no evidence of bleeding, and the patient recommenced lenvatinib treatment 14 days after the intravascular treatment. One week after lenvatinib resumption, contrast-enhanced CT showed a decrease in the liver tumor enhancement, and the lung metastases rapidly decreased.

Lenvatinib is an inhibitor of multiple receptor tyrosine kinases and is widely used as the first-line treatment for unresectable HCC. Hepatocellular carcinoma rupture related to lenvatinib treatment appears to be extremely rare [1], and

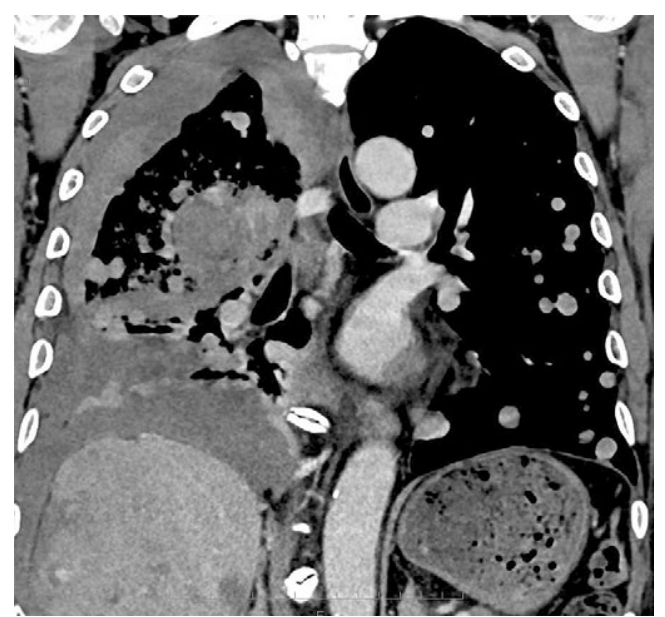

Fig. 1. Contrast-enhanced computed tomography showing a metastatic tumor rupture in the right lung.

no case of rupture of distant metastasis from HCC associated with lenvatinib has been reported. The mechanism of HCC rupture associated with lenvatinib therapy has not been clarified yet but may be explained by rapid tumor shrinkage [2], rapid partial tumor necrosis [3], and/or rapid ischemic change [4]. Lenvatinib treatment resulted in an early and pronounced reduction in tumor size in the first 2 months in thyroid cancer, although it is unclear whether the response might have been even more rapid [5]. Actually, lenvatinibassociated HCC ruptures occurred within one week after initiating therapy [1] as observed in the present case. It should be considered that lenvatinib might cause rupture of HCC, including distant metastasis from HCC, in the early phase of lenvatinib treatment.

Masayuki Higashino ${ }^{1}$, Ryo Sugiura ${ }^{1,2}$, Yoshiya Yamamoto $^{1}$, Hirohito Naruse $^{1}$, Naoya Sakamoto ${ }^{2}$

1) Department of Gastroenterology and Hepatology, Hakodate Municipal Hospital, Hakodate; 2) Department of Gastroenterology and Hepatology, Hokkaido University Faculty of Medicine and Graduate School of Medicine, Sapporo, Japan

Correspondence: Ryo Sugiura, MD, PhD, ryou99sugi@yahoo.co.jp

\section{Conflicts of interest: None.}

Acknowledgements: We express our deepest appreciation to Dr. Kazuteru Hatanaka, Jun Ito, Kenji Kinoshita, Shuichi Miyamoto, Shuhei Hayasaka and Naohisa Tsuchida (Department of Gastroenterology and Hepatology, Hakodate Municipal Hospital) for clinical advice.

DOI: $10.15403 /$ jgld-3025

\section{REFERENCES}

1. Kotani K, Uchida-Kobayashi S, Yoshida K, et al. Lenvatinibinduced tumor-related hemorrhage in patients with unresectable hepatocellular carcinoma. Am J Gastroenterol 2020. doi:10.14309/ ajg.0000000000000747

2. Date E, Okamoto K, Fumita S, Kaneda H. Gastrointestinal perforation related to lenvatinib, an anti-angiogenic inhibitor that targets multiple 
receptor tyrosine kinases, in a patient with metastatic thyroid cancer. Invest New Drugs 2018;36:350-353. doi:10.1007/s10637-017-0522-4

3. Suzuki N, Tajiri K, Futsukaichi Y, et al. Perforation of the small intestine after introduction of lenvatinib in a patient with advanced hepatocellular carcinoma. Case Rep Gastroenterol 2020;14:63-69. doi: $10.1159 / 000505774$

4. Takeda H, Nishijima N, Nasu A, et al. Long-term antitumor effect of lenvatinib on unresectable hepatocellular carcinoma with portal vein invasion. Hepatol Res 2019;49:594-599. doi:10.1111/hepr.13294

5. Robinson B, Schlumberger M, Wirth LJ, et al. Characterization of tumor size changes over time from the phase 3 study of lenvatinib in thyroid cancer. J Clin Endocrinol Metab 2016;101:4103-4109. doi:10.1210/ jc.2015-3989

\section{Normal histology, the new target for ulcerative colitis treatment, might not be the cure}

\section{To the Editor,}

We read with great interest the article written by Manuc et al. [1] regarding the molecular signature of persistent histological inflammation in ulcerative colitis (UC) with mucosal healing. We agree that the endoscopic evaluation has a poor correlation with the histologic inflammatory activity, rendering this method of clinical assessment less than ideal [2].

Although the Geboes score is only a partially validated index and the histologic remission might be a goal hard to reach, there are data proving higher rates of relapse in patients with any residual inflammatory activity (up to $57.69 \%$ rate of relapse even in patients with quiescent histological activity). $[3,4]$. The differentiation between quiescence activity and normal histology is thus, of great importance [5]. The optimal biopsy sampling density has not yet been established, but the actual recommendation is to take two biopsies from each colonic segment using a large capacity forceps within at least 2 moments [2]. This measure will help to distinguish if the histologic normalization is transient or may have been a sampling or interpretation error.

In the study conducted by Manuc et al. [1], patients were biopsied during different time points, but only biopsies from the sigmoid colon were analyzed, even in patients with disease extended beyond the splenic flexure (E3). The high number of target genes studied offered the chance to identify 29 positive correlations with the Geboes score. Having a quantifiable genetical analysis of the histological activity is important in guiding treatment decisions accurately and precisely.

Our team prospectively followed 25 patients ( $60 \%$ male) with UC between January 2012 and January 2016 who underwent medical de-escalation therapy due to normal histology proved in rectal biopsies. Patients in clinical, endoscopic and histologic remission were followed up to May 2020. All patients were treated with anti tumor necrosis factor (TNF) alfa with 2 subsequent evaluations proving histological normalization of the colorectum. The median age at UC diagnosis was $28+/$ 14.8 years. The disease distribution by Montreal classification was 17 (68\%) E3 and 8 (32\%) disease extended till the splenic flexure (E2). The median time to reach bio-clinical, endoscopic and histological remission was 18, 36 and 69 weeks. All patients relapsed and the median time to clinical, endoscopic and histological relapse was 23,11, and 6 months. Most of them $(20.8 \%)$ responded to reinduction therapy with the same anti TNF-alfa drug or alternative therapy.

Formyl-peptide receptor 1 (FPR1), matrix metalloproteinases 1 (MMP1) and mucine 1 (MUC1) proved to be in correlation with the clinical course and histological activity, providing their utility as new prognostic markers in IBD [1]. A complete characterization of gene expression profile will identify a new clinical assessment of phenotype in patients with IBD. These data proved that a single finding of histological remission (probably normal histology) was associated with a good prognosis, but the risk to further relapses might be strongly influenced and directed by the genetic profile of each patient and the type of pathological mechanism causing the disease.

Cristian Gheorghe ${ }^{1,2}$, Anca Dimitriu ${ }^{1,2}$, Roxana Costache ${ }^{1}$, Monica Cojocaru $^{1,2}$, Liana Gheorghe ${ }^{1,2}$

1) Center of Gastroenterology and Hepatology, Fundeni Clinical Institute, Bucharest; 2) Carol Davila University of Medicine and Pharmacy, Bucharest, Romania

Correspondence: Anca Dimitriu, Borcaanca@yahoo.com

Conflicts of interest: None.

DOI: $10.15403 /$ jgld-2927

\section{REFERENCES}

1. Manuc M, Ionescu EM, Milanesi E, et al. Molecular Signature of Persistent Histological Inflammation in Ulcerative Colitis with Mucosal Healing. J Gastrointestin Liver Dis 2020;29:159-166. doi:10.15403/ JGLD-576

2. Chateau T, Feakins R, Marchal-Bressenot A, Magro F, Danese S, Peyrin-Biroulet L. Histological Remission in Ulcerative Colitis: under the microscopie is the cure. Am J Gastroenterol 2020;115:179-189. doi:10.14309/ajg.0000000000000437

3. Camarillo GF, Goyon EI, Zuñiga RB, et al. Gene Expression profiling of mediators associated with the inflammatory pathways in the intestinal tissue from patients with ulcerative colitis. Mediators Inflamm 2020;2020:9238970. doi:10.1155/2020/9238970

4. Azad S, Sood N, Sood A. Biological and Histological Parameters as Predictors of Relapse in Ulcerative Colitis: A Prospective Study. Saudi J Gastroenterol 2011;17:194-198. doi:10.4103/1319-3767.80383

5. Israel A, Christensen B, El Jurdi K, et al. Follow-up ormalizationof patients with ulcerative colitis and histological normalisation. Clin Gastroenterol Hepatol 2020;18:987-988. doi:10.1016/j. cgh.2019.06.025

\section{Nurses may be safely involved in sedation for colonoscopy}

\section{To the Editor,}

As a nurse myself and as President of ENDOGAST, the Romanian Society of Endoscopy and Gastroenterology Nurses, a member of ESGENA (The European Society of 
Gastroenterology and Endoscopy Nurses and Associate), may I thank you for publishing in your most recent issue the paper "Nurse Administered Propofol Sedation (NAPS) versus On-call Anesthesiologist Administered Propofol Sedation (OAPS) in Elective Colonoscopy" by Tiankanon et al. [1] This is an interesting study from Thailand showing that nurses may prepare sedation with propofol for colonoscopy. The authors tried to find out if the easier and cheaper procedure to use trained nurses for colonoscopy sedation can replace on-call anesthesiologists. In a retrospective survey, the authors concluded that elective colonoscopy with sedation performed by nurses is as successful in low-risk patients, as colonoscopy using anesthesiologists for sedation. However, the dose of propofol was lower when administered by nurses and accordingly, the adverse effects were scarcer [2]. These data suggest that the sedation by nurses is milder, probably because of prudence from the part of endoscopists and nurses. The nurses in this study were endoscopy nurses with special training in propofol sedation, and not anesthesia nurses. Therefore, we consider that this model should be extended to other areas, such as ours. Indeed, in this country, more emphasis should be put not only on the training of endoscopy and gastroenterology nurses, but also on their ability to offer sedation for endoscopies.

The results should be compared with data provided by the almost simultaneously published paper by McKenzie et al. [2]. This is another retrospective study from Utah, USA. The authors analyzed the outcomes of a large sample of almost 19,000 colonoscopies in which nurses performed anesthesia in low-risk cases compared to high-risk patients. Stratification of severity was carried out according to the American Society of Anesthesia (ASA) I-III criteria. The study showed that the safety of high-risk colonoscopies (ASA III) is similar to those of low-risk patients (ASA I and II).

The results of both papers are consistent with those reported by another study, on a larger group of patients with low-risk [3]. This paper also concluded that nurses in endoscopy units are able to perform safe and efficient sedation for colonoscopy, as well as for other endoscopic investigations.

The use of sedation by nurses is not new in colonoscopy [4] but the advantages of these procedure needed to be analyzed and evaluated. Later, and based on the accumulation of experience, the procedure was even recommended in the guidelines of ESGENA [5]. However, in our country the sedation remains the task of anesthesiologists, to avoid adverse effects and associated forensic pursuits.

On behalf of my colleagues, may I thank you for continuing the publication of papers on nurses' activities in endoscopy, which displays their efficiency, such as in the paper by Guarini et al. [6] on the ability of nurses to read and evaluate video capsule endoscopic recordings. Thus, these papers prove that nurses fulfill their mission to assist gastroenterologists in their work.

\section{Liliana David}

Department of Nursing, Iuliu Hatieganu University of Medicine and Pharmacy Cluj-Napoca, Romania

Correspondence: Liliana David, Liliana.David@umfcluj.ro
Conflicts of interest: None.

DOI: 10.15403/jgld-3353

\section{REFERENCES}

1. Manuc M, Ionescu EM, Milanesi E, et al. Molecular Signature of Persistent Histological Inflammation in Ulcerative Colitis with Mucosal Healing. J Gastrointestin Liver Dis 2020;29:159-166. doi:10.15403/ JGLD-576

2. Chateau T, Feakins R, Marchal-Bressenot A, Magro F, Danese S, Peyrin-Biroulet L. Histological Remission in Ulcerative Colitis: under the microscopie is the cure. Am J Gastroenterol 2020;115:179-189. doi:10.14309/ajg.0000000000000437

3. Camarillo GF, Goyon EI, Zuñiga RB, et al. Gene Expression profiling of mediators associated with the inflammatory pathways in the intestinal tissue from patients with ulcerative colitis. Mediators Inflamm 2020;2020:9238970. doi:10.1155/2020/9238970

4. Azad S, Sood N, Sood A. Biological and Histological Parameters as Predictors of Relapse in Ulcerative Colitis: A Prospective Study. Saudi J Gastroenterol 2011;17:194-198. doi:10.4103/1319-3767.80383

5. Israel A, Christensen B, El Jurdi K, et al. Follow-up ormalizationof patients with ulcerative colitis and histological normalisation. Clin Gastroenterol Hepatol 2020;18:987-988. doi:10.1016/j.cgh.2019.06.025

\section{Hepatic sinusoidal obstruction syndrome associated with nivolumab: an uncommon adverse event related to immune checkpoint inhibitors}

\section{To the Editor,}

Oncological therapies based on immune checkpoint inhibitors (CPI) have shown a remarkable benefit in many tumour types [1]. However, these treatments are also associated with adverse events. We report the case of a patient suffering from a melanoma treated with nivolumab who developed portal hypertension secondary to hepatic sinusoidal obstruction syndrome (SOS) as a rare immune-related adverse event.

A 67-year-old man without history of alcohol intake, was admitted to hospital for fever and ascites. His medical background included arterial hypertension, ischemic heart disease and resected lumbar cutaneous melanoma (stage IIIC) treated with adjuvant immunotherapy with an anti-PD1 (nivolumab) until June 2018, achieving no evidence of disease. One month after completing immunotherapy he received treatment with corticoids for a grade 3 aspartate aminotransferase (AST) increase. At that moment, blood tests showed AST of 288 $\mathrm{UI} / \mathrm{L}$ (8-50 IU/mL), alanine aminotransferase (ALT) $120 \mathrm{IU} / \mathrm{L}$, gamma glutamyl-transferase $143 \mathrm{IU} / \mathrm{L}$ (9-55 IU/L), alkaline phosphatase $97 \mathrm{UI} / \mathrm{L}(30-120 \mathrm{IU} / \mathrm{L})$ and total bilirubin $1.6 \mathrm{mg} /$ $\mathrm{dL}$ (0.3-1.2 mg/dL). A positron emission tomography (PET/CT) scan ruled out a relapse of melanoma and an ultrasound showed a liver with normal size, normal biliary tree and permeable hepatic and portal veins. Tests for viral hepatitis were negative and anti-nuclear antibodies (ANAs) tested positive at titres of 1:320. Corticoids at dose of $1 \mathrm{mg} / \mathrm{kg}$ per day were initiated as an immune-related hepatitis was suspected. Response was good with normalization of AST levels. 

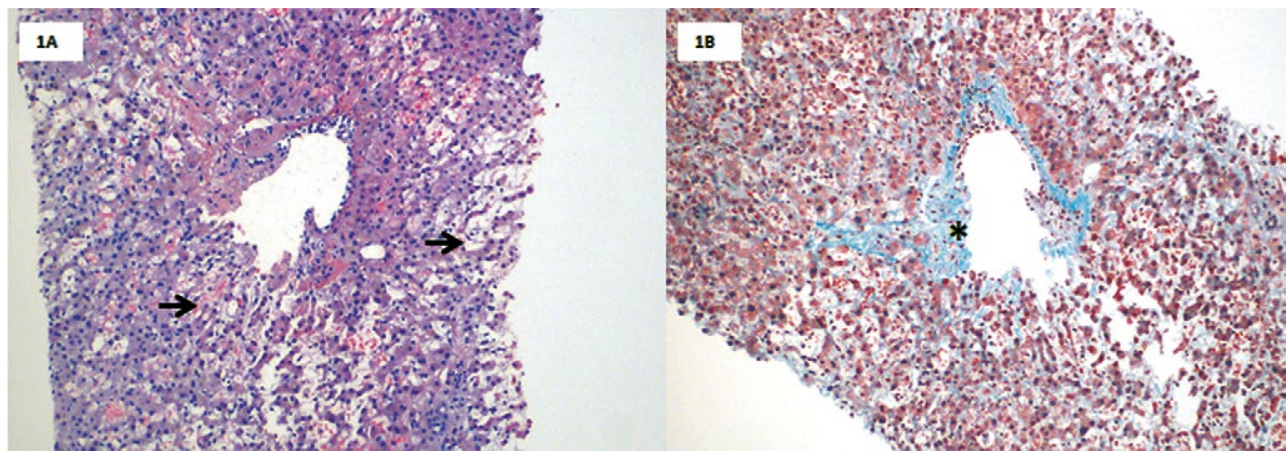

Fig. 1. Liver biopsy 2018. Panel A, H\&E, 100x: Venous partial occlusion of the centrilobular hepatic venous branch with mild lymphocytic inflammation, marked dilation (à) and sinusoidal blood stasis and trabecular atrophy. Panel B, Tricromic stain, 100x: edema and lax subendothelial fibrous proliferation, without evidence of sinusoidal fibrosis $\left(^{*}\right)$.

Despite antibiotic prophylaxis with co-trimoxale, three weeks after the immune-related hepatitis the patient was admitted to hospital due to fever and dyspnea. Physical examination showed bibasilar rales and moderate ascites. He was diagnosed with acute respiratory failure due to staphylococcus aureus methicillin-resistant bilateral pneumonia. He developed a septic shock requiring ventilator support and vasoconstrictor drugs. During his hospital stay, clinical signs of portal hypertension in the form of ascites were observed. Ascitic fluid was a transudate with low cellularity and lactate dehydrogenase levels and normal glucose. A liver biopsy showed images of veno-occlusive disease (Fig. 1). Liver fibrosis or malignancy were excluded. The hepatic venous pressure gradient (HVPG) was $25 \mathrm{mmHg}$. Initially, the possibility of a transjugular intrahepatic portosystemic shunt (TIPS) was considered, but as a consequence of the septic shock the patient developed kidney injury that required renal replacement therapy which successfully controlled the ascites.

Therapy with CPI can lead to dysimmune toxicities, named as immune-related adverse events that can potentially affect any organ at any time during or even after treatment has ended [2]. Immune-related hepatitis is usually presented as asymptomatic AST or ALT increase, though other more uncommon manifestations have been reported such as nodular regenerative hyperplasia [3]. Hepatic sinusoidal obstruction syndrome (SOS) also known as veno-oclussive disease, is characterized by obstruction to the hepatic venous flow by occlusion of the terminal hepatic venules and hepatic sinusoids, conditioning a postsinusoidal portal hypertension. It has been suggested that some drugs can induce a diffuse T-cell infiltrate in the hepatocytes, prominent sinusoidal histiocytic infiltrates and central vein damage with endotheliitis/endothelial injury. Accordingly, the histology of patients with immune-related hepatitis revealed central vein endotheliitis in 2 out of 9 patients treated with an anti-PD-1/L1 [4]. Furthermore, an isolated report of SOS associated with nivolumab has been described [5]. In our clinical case, due to the development of chronic kidney disease as a consequence of the infectious complications, the patient required renal replacement with haemodialysis which successfully controlled the ascites, with no need of TIPS.

In summary, sinusoidal obstruction syndrome could be associated with CPI which is a complication that widens the spectrum of adverse events linked to immunotherapy. This report highlights the need to rule out sinusoidal obstruction syndrome in patients who develop ascites while or after undergoing immunotherapy.

\section{Mar Riveiro-Barciela ${ }^{1,2}$, Didac González-Sans ${ }^{1}$, David Marmolejo ${ }^{3}$, Maria-Teresa Salcedo ${ }^{4}$, Eva Muñoz-Couselo ${ }^{3}$}

1) Liver Unit, Department of Internal Medicine, Hospital Universitari Vall d'Hebron, Vall d'Hebron Barcelona Hospital Campus and Universitat Autònoma de Barcelona, Barcelona; 2) Centro de Investigación Biomédica en Red de Enfermedades Hepáticas y Digestivas (CIBERehd), Instituto de Salud Carlos III, Madrid; 3) Oncology Department, Vall d'Hebron Institut of Oncology (VHIO), Hospital Universitari Vall d'Hebron, Vall d'Hebron Barcelona Hospital Campus, Barcelona; 4) Pathology Department, Hospital Universitari Vall d'Hebron, Vall d'Hebron Barcelona Hospital Campus, Barcelona, Spain

Correspondence: Mar Riveiro-Barciela, mar.riveiro@gmail.com

\section{Conflicts of interest: None.}

Acknowledgments: English writing support was provided by Fidelma Greaves.

DOI: $10.15403 /$ jgld-3207

\section{REFERENCES}

1. Gubin MM, Zhang X, Schuster H, et al. Checkpoint blockade cancer immunotherapy targets tumour-specific mutant antigens. Nature 2014;515:577-581. doi:10.1038/nature13988

2. Postow MA, Sidlow R, Hellmann MD. Immune-Related Adverse Events Associated with Immune Checkpoint Blockade. N Engl J Med 2018;378:158-168. doi:10.1056/NEJMra1703481

3. LoPiccolo J, Brener MI, Oshima K, Lipson EJ, Hamilton JP. Nodular Regenerative Hyperplasia Associated With Immune Checkpoint Blockade. Hepatology 2018;68:2431-2433. doi:10.1002/hep.30157

4. De Martin E, Michot JM, Papouin B, et al. Characterization of liver injury induced by cancer immunotherapy using immune checkpoint inhibitors. doi:10.1016/j.jhep.2018.01.033

5. Charvet E, Lheure C, Isnard C, et al. Hepatic sinusoidal obstruction syndrome induced by nivolumab in advanced melanoma: a case report. Ann Oncol 2020;31:661-662. doi:10.1016/j.annonc.2020.02.004 


\section{Tranexamic acid retention for gallbladder bleeding}

\section{To the Editor,}

Bleeding within gallbladders is a rare clinical presentation, even in uremic patients [1]. Gallbladder bleeding may result from trauma, biliary neoplasms, acute infectious cholecystitis, cirrhosis, renal failure, and the use of anticoagulants. Surgery is the treatment of choice for hemorrhagic gallbladder [1], but cholecystectomy may not be suitable for hemodynamically unstable patients. The reason why uremic patients, whether on hemodialysis (HD) or not, have a higher risk of bleeding is multifactorial. Erythropoiesis-stimulating agents, desmopressin, estrogen, and cryoprecipitate can be used in treating hemorrhage in uremic patients [2]. In uremic patients, systemic tranexamic acid (TXA) should be administrated with caution due to possible seizures [3]. Herein, we reported a successful experience of treating gallbladder bleeding by TXA retention via the percutaneous transhepatic gallbladder drainage (PTGBD) tube in an HD woman.

An 87-year-old female patient had gallstones, type 2 diabetes mellitus, and end-stage kidney disease on HD thrice weekly. A PTGBD tube had been placed for her relapsing cholecystitis. She used a high-flux dialyzer and received darbepoetin alfa 20 mcg twice a week. Severe hemorrhage in the gallbladder with bloody bile from the PTGBD tube developed after a traumatic pulling-out episode. The abdominal computed tomography scan confirmed the tube was still in the gallbladder lumen. Her hemoglobin was $10.5 \mathrm{mg} / \mathrm{dL}$, predialysis serum creatinine 4.5-5.5 mg/dL, C-reactive protein $0.81 \mathrm{mg} / \mathrm{dL}$, and INR 0.91.05. We had optimized the uremic bleeding management by intensive desmopressin, estrogen, and transfusion of packed red blood cells, cryoprecipitate, and platelets. Parenteral vitamin K supplement was also given for the reduced absorption of fatsoluble vitamins caused by bile loss. However, the bloody bile persisted. Due to multiple morbidities, cholecystectomy was not suggested. Therefore, one $\mathrm{mL}$ of TXA $50 \mathrm{mg} / \mathrm{mL}$ in normal saline $9 \mathrm{~mL}$ was instilled into the gallbladder via the PTGBD tube. The tube was clamped for 30 minutes. She did not receive any treatment for uremic bleeding thereafter. The bloody bile subsided entirely, five days after the procedure.

Tranexamic acid, a synthetic lysine analog, has a strong anti-fibrinolytic effect and has been widely used in prophylaxis and treatment of bleedings [4]. A meta-analysis concluded that intravenous TXA did reduce the requirement of blood transfusion during multiple surgeries. Nevertheless, the potential sequelae such as stroke, pulmonary embolism, deep vein thrombosis, myocardial infarction, and seizures could be fatal [5]. Thus, the safety and effectiveness of local applications of TXA for stopping bleeding had been researched. A metaanalysis reported that in orthopedic surgeries, topical TXA was as effective as its intravenous use in reducing transfusion [3].

Recently, a double-blinded randomized controlled trial was performed in patients with gross hematuria [4]. All the patients received three-way urinary catheter insertion and normal saline irrigation. In the study group, TXA $500 \mathrm{mg}$ in $100 \mathrm{~mL}$ of normal saline was injected via the catheter. The catheters were clamped to stop the urinary flow for 15 minutes. There was a statistically significant reduction of the irrigated normal saline volumes in the TXA group.

Our patient was at a very high risk for general anesthesia and surgical intervention. Her bloody bile persisted despite optimizing HD efficacy and aggressive management for uremic bleeding. Therefore, based on the results of the pilot trial described above, we retained TXA through the established PTGBD tube. The bile became completely clear five days later, without any adverse effects. To our knowledge, this is the first report describing the retention of TXA in a PTGBD tube to stop gallbladder bleeding. Our report provides a useful method, and more research regarding the intra-catheter injection of TXA for gallbladder bleeding is required.

\section{Wen-Chi Wu ${ }^{1,2}$, Der-Cherng Tarng ${ }^{3,4,5,6}$, Chih-Yu Yang ${ }^{3,4,6,7}$}

1) Division of Medical Oncology, Department of Oncology, Taipei Veterans General Hospital, Taipei; 2) Division of Hematology, Department of Medicine, Taipei Veterans General Hospital, Taipei; 3) Division of Nephrology, Taipei Veterans General Hospital, Taipei; 4) Institute of Clinical Medicine, School of Medicine, National YangMing University, Taipei; 5) Department and Institute of Physiology, National Yang-Ming University, Taipei; 6) Center for Intelligent Drug Systems and Smart Bio-devices (IDS2B); 7) Stem Cell Research Center, National Yang-Ming University, Taipei, Taiwan

Correspondence: Chih-Yu Yang, MD, PhD, cyyang3@vghtpe.gov.tw

\section{Conflicts of interest: None}

Acknowledgments: This work was in part supported financially for research purposes by the "Yin Yen-Liang Development and Construction Plan" of the School of Medicine, National Yang-Ming University, Taipei, Taiwan (107F-M01-0504), the Ministry of Science and Technology, Taipei, Taiwan (MOST 109-2314-B-010-053-MY3 and MOST 109-2321-B-009-007), Taipei Veterans General Hospital, Taipei, Taiwan (VGHUST107-G5-3-3 and VGHUST109-V5-1-2), and the "Center for Intelligent Drug Systems and Smart Bio-devices (IDS2B)" from The Featured Areas Research Center Program, MOE in Taiwan. The funders have no role in the study design, data collection, analysis, and interpretation, or in the writing of the manuscript.

DOI: $10.15403 /$ jgld-3078

\section{REFERENCES}

1. Parekh J, Corvera CU. Hemorrhagic cholecystitis. Arch Surg 2010;145:202-204. doi:10.1001/archsurg.2009.265

2. Hedges SJ, Dehoney SB, Hooper JS, Amanzadeh J, Busti AJ. Evidencebased treatment recommendations for uremic bleeding. Nat Clin Pract Nephrol 2007;3:138-153. doi:10.1038/ncpneph0421

3. Montroy J, Hutton B, Moodley P, et al. The efficacy and safety of topical tranexamic acid: A systematic review and meta-analysis. Transfus Med Rev 2018;31:165-178. doi:10.1016/j.tmrv.2018.02.003

4. Moharamzadeh P, Ojaghihaghighi S, Amjadi M, Rahmani F, Farjamnia A. Effect of tranexamic acid on gross hematuria: A pilot randomized clinical trial study. Am J Emerg Med 2017;35:1922-1925. doi:10.1016/j. ajem.2017.09.012

5. Ker K, Edwards P, Perel P, Shakur H, Roberts I. Effect of tranexamic acid on surgical bleeding: systematic review and cumulative meta-analysis. BMJ 2012;344:e3054. doi:10.1136/bmj.e3054 